\title{
Correlative study of the emission from flares associated with Sun quakes
}

\author{
J. C. Martínez-Oliveros, A.-C. Donea and P. S. Cally \\ Centre for Stellar and Planetary Astrophysics, School of Mathematical Sciences \\ Monash University, VIC 3800, Australia \\ email:Juan.Oliveros@sci.monash.edu.au
}

\begin{abstract}
Multi-wavelength studies of energetic solar flares with seismic emissions have revealed interesting common features that may help us to identify the correlations of flare signatures from the inner to the outer solar atmosphere and, to develop diagnostic techniques to aid in the sun quake detection. In our study, we make use the relation between the microwave and the hard X-ray emissions associated with such flares to propose a scenario for the ignition of seismic transients from flares. We explore the mechanisms of energy transport to the photosphere, such us back-warming or direct particle impacts.
\end{abstract}

Keywords. Sun: X-rays, Sun: particle acceleration, Sun: helioseismology, Sun: sun quakes, Sun: solar flares

\section{Introduction}

From the first discovery of a sun quake by Kosovichev \& Zharkova (1998) many other works had been written about the detection of flare generated seismic waves in the Sun (Kosovichev 2006; Zharkova \& Zharkov 207; Donea, Braun \& Lindsey 1999; Donea \& Lindsey 2005; Donea et al. 2006; Besliu-Ionescu et al. 2006; Moradi et al. 2007; MartínezOliveros et al. 2007a,b). The sun quakes are defined the observed expanding waves on the solar photosphere induced by high energy particles (electrons and/or protons) accelerated by the flare. It is believe that sun quakes are produce by the momentum or energy transfer by high energy particles into the solar photosphere. This momentum will induce the generation of an internal pressure wave that travel in solar interior and eventually will reach again the surface after a determined length of time. This is observed as a close to circular change in the mean velocity amplitude of the photosphere in the MDI-Dopplerograms which resambles ripples propagating from the place where particles hit the lower chromosphere. Depending of the initial impact velocity the wave will travel less or deeper into the solar interior, reaching the surface at different times. The overall picture of this process is the apparent motion of the surface wave.

As the importance of the particles (electrons and/or protons) interaction with the solar atmosphere has been already revealed (Zharkova \& Zharkov 207; Donea et al. 2006; Moradi et al. 2007; Martínez-Oliveros et al. 2007a), the study of their acceleration, propagation and precipitation is a key area in the research of the solar seismicity. The solar model divided the flare process in several stages: acceleration, injection, propagation, trapping, precipitation and energy losses. Each of these stages have a distinctive type of emission and timing associated with the physical processes ocurring at that time. Therefore, studying of the flare emission at different wavelengths can give us important information about the physical conditions under which a sun quake can be generated.

In our discussion the mechanism of acceleration and injection of particles in the coronal magnetic field from the acceleration region will be considered a black box. After particles have been injected into the coronal magnetic field they begin to move along the 
magnetic field lines. This depends on several factors such as the initial pitch angle and injection speed. The initial population of electrons can be split into a precipitated and a trapped populations. These populations have specific type of electromagnetic emissions that can be determined. The trapped population can be mainly observed in the microwave frequency band as produced by the electrons gyrating along the magnetic field lines, whereas the precipitating population of electrons signal in hard X-rays as they collide with the dense chromosphere. The electrons trapped in the magnetic field loop will eventually precipitate in the lower chromosphere or wiil be thermalized in the corona as they collide with the ambient plasma. It is important to remark that the absence of a thermal decay in the microwave flux of the acousticaly active flares has been noticed by Martínez-Oliveros et al. (2007a,b) and is in agreement with the dynamical trapping model of Aschawanden (2004). In this model the density of the trapped population is conditioned to the relaxation of the coronal magnetic field lines. The aperture angle of the loss cone increases with time as the coronal loop tend to a more potential configuration low in altitude.

In this work we describe a multi-wavelength observational technique that may help to identify the seismicity level of a flare. Two seismically actively solar flares have been found using this method. We define the objectives of our work as follows:

- Establish an empirical relation between fluxes at different wavelengths.

- Study the trapped and precipitated particle populations, using observations in microwaves (MW) and hard X-rays (HXR).

- Study the spatial and temporal correlation between observed sources.

- Establish a relation between the morphology of the seismic source and the physical properties of the energy deposition mechanism in flares.

\section{Observations and Results}

We restrict our search to those GOES X-class flares that have already been associated with seismic events (see http://www.maths.monash.edu.au/dbesliu/sun quakes/sun quakes.html). From this sample we selected those observed simultaneously by RHESSI and the Nobeyama Radio Heliograph (NoRH), as we are interested to study the trapped and precipitated populations of non-thermal particles.

The seismic sources were detected using the computational seismic holography (Lindsey \& Braun 2000). The location of the seismic source is then campared with the position of the sources at different wavelengths and specially with the magnetic and Doppler transients, white light sources and HXR footpoints. We found that all sources are spatially well correlated. Their temporal behaviour was also studied and we confirm previous observations (Donea, Braun \& Lindsey 1999; Donea \& Lindsey 2005; Donea et al. 2006; Besliu-Ionescu et al. 2006; Moradi et al. 2007; Kosovichev \& Zharkova 1998). Studing the MW emission of the flare we noticed the almost total absence of a long term thermal decay, that characterizes the non-impulsive events, suggesting that only highly impulsive flares are capable to generate some sort of seismic response of the photosphere.

The magnetic field configuration of the flaring active region was studied using SOHO/MDI magnetograms and vectormagnetograms to recreate potential and non-linear coronal magnetic field lines. From the extrapolations we found that, for the flare with the GOES class between M9.5 and X5, the magnetic field configuration of the flaring region was composed by highly sheared low lying magnetic loops. These results were compared with EUV TRACE images to show that the magnetic calculations were close to the real configuration of the magnetic field in the active regions prior to the flare.

Using these first observational results we searched for flares shearing the same emission properties in MW and HXR with a high sheared low-lying magnetic field configuration. 
We have chosen two flares to study: the 14 August 2004 M7.4 solar flare and the 10 March 2001 M6.7 solar flare.

\subsection{August 2004 M7.4 solar flare}

The 14 August 2004 M7.4 solar flare hosted by the active region 10656, beginning at 05:36 UT, peaking at 05:44 UT and ending at 05:52 UT. It is important to remark that this active region hosted two sun quakes within a period of 48 hours: the first generated on 13 of August 2004 by an X1.0 solar flare and the second on 15 August 2004 produced by a M9.5 solar flare (Besliu-Ionescu et al. 2006; Donea et al. 2006).
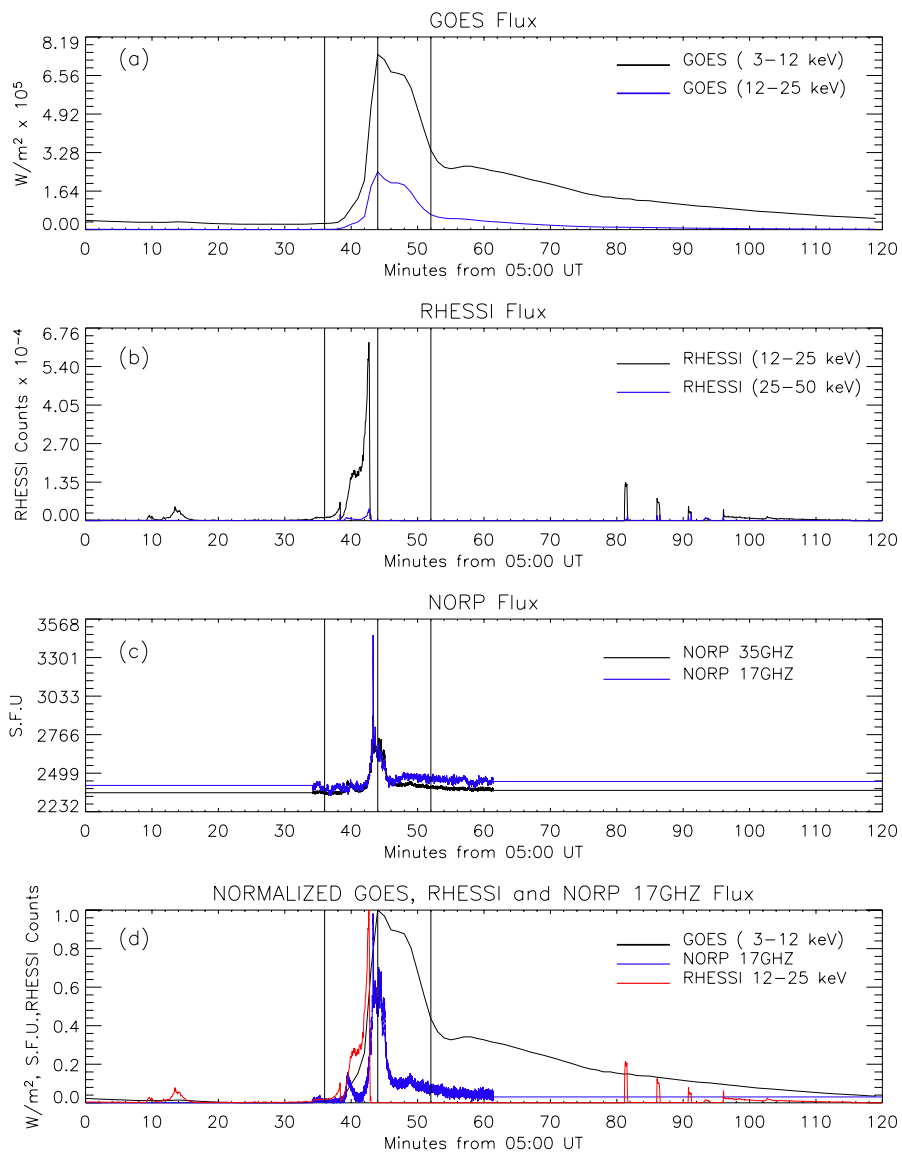

Figure 1. Total flux time profiles for the 14 August 2004 solar flare: (a) The GOES total flux in the two channels $1-8 \AA$ (black line) and $0.5-4 \AA$ (blue line), (b) HXR time profile from RHESSI in the two channels $15-25 \mathrm{keV}$ (black line) and $25-50 \mathrm{keV}$ (blue line), (c) shows the microwave time profiles from NoRP data at $17 \mathrm{GHz}$ (blue line) and $35 \mathrm{GHz}$ (black line), (d) Normalized plot of the GOES total flux at 1-8 $\AA$ (black line), RHESSI $12-25 \mathrm{keV}$ (red line) and the NoRP flux at $17 \mathrm{GHz}$ (blue line).

The microwave emission tell us what is the energy contribution of the trapped electrons gyrating in the coronal magnetic field. The lightcurve has two different components: a dominant intensity pulse and an intensity decay of the microwave emission. The rise of the first component is well correlated with the rise of the HXR emission. This is because the same electronic populations are responsible for both emissions (Bastian et al. 1998). The frst MW peak is produced by a ppopulation of electrons directly injected 
into the lower chromosphere. The decay in MW intensity is releated to the motion of the trapped electrons in the coronal magnetic loop. We beleive that the first electronic population which gives the sudden character of the MW time series is also responsible for the generation of seismic waves in the photosphere.

In the Figure 1 we observe that the flux of microwave emission from the second electronic population trapped in the magnetic loop decays in a short period of time, leaving less that $10 \%$ of the total MW flux decaying slowly. This indicates that the injection mechanism was effective, thus the electrons trapped in the coronal magnetic field were rapidly scattered within the loss cone. Another explanation of this observation is that the thermalization of the electrons happened in a region optically thick for the microwaves.

The measured brightness temperature of the radio source is $4.67 \times 10^{7} \mathrm{~K}$, and the computed spectral emission index was $\delta=-3.67$ (Martínez-Oliveros et al. 2007a). These parameters suggest that a non-thermal emission process is responsible for the microwave radiation. Using a variation technique for solar radio images analysis (Grechnev 2003) we have found that the non-thermal emission region associated with the electron injection region in the flare. This region appears to be spatially well correlated with the HXR emission prior to the maximum MW emission.
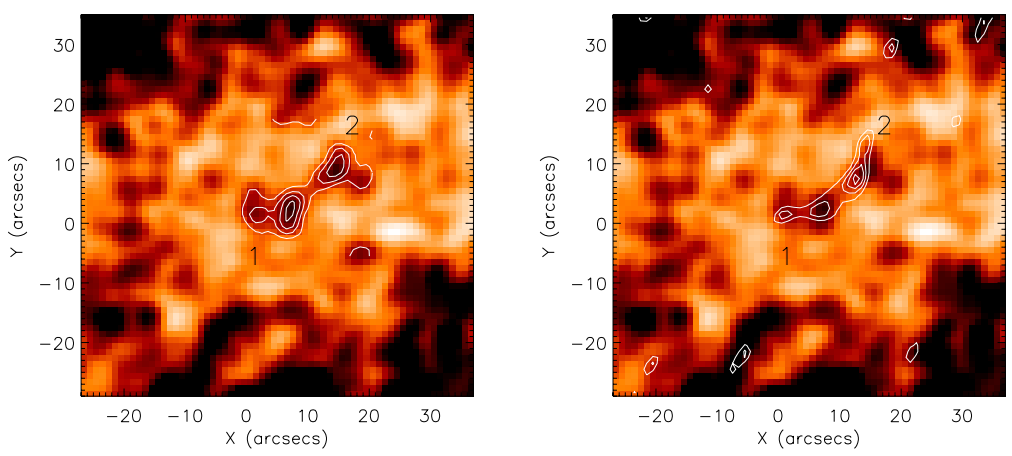

Figure 2. Left: $6 \mathrm{mHz}$ egression power snapshot at 05:42 UT of the acoustic kernel sources of the 14 August 2004 seismic emission, with contour levels of $50 \%, 65 \%, 80 \%$ and $95 \%$ of the maximum source intensity. Right: Egression power map and RHESSI contour plots, with levels of $30 \%, 50 \%, 70 \%$ and $90 \%$ of the maximum source intensity.

We have also analysed the temporal and spatial evolution of the RHESSI HXR source. The source moves in the North-East direction. This motion appears to be diagonal to the magnetic neutral line. Also we noticed a change in the morphology of the X-ray source, beginning as a compact object and endding as an extended object crossing the neutral line. This elongated HXR source is composed mainly of two kernels, located at opposite magnetic polarities that could be the two footpoints of the same loop.

The above observations lead us to the conclusion that the M7.4 flare must have produced a sun quake. We apply two different but in our opinion complementary techniques to reveal the seismicity of the active region: the holographic seismology and the timedistance plot analysis. We generated the time-distance plot for this region over azimuths $-50^{\circ}$ and $+20^{\circ}$ and we have detected a weak ridge that can be associated with an expanding seismic wave. The computational seismic holography (Lindsey \& Braun 2000) confirmed our thoughts. We have located the acoustic source generated by this flare on the egression power maps at $6 \mathrm{mHz}$. Comparing the $6 \mathrm{mHz}$ egression power snapshot of the seismic source at 05:42 UT with the HXR 12-25 keV source (Figure 2), we found that both sources are spatially well correlated and with a similar morphology. 
2.2. 10 March 2001 M6.7 solar flare

Using the same technique we have studied the emission properties if the 10 March 2001 M6.7 solar flare. This flare was very well observed and studied by many authors Liu, Ding \& Fang (2001); Ding et al. (2003); Uddin et al. (2004); Li, Ding \& Liu (2005). The flare began at 04:00 UT, reaching its maxima at 04:05 UT and ending at 04:07 UT in the active region 9368. Figure 3 shows that the time profile in MW does not present the exponential decay that could be associated with the thermalization of trapped electrons in the corona. The Yohkoh HXR emission in the M2 and $\mathrm{H}$ channels shows the precipitation of high energy electrons into the chromosphere occurs in a relatively short period of time.
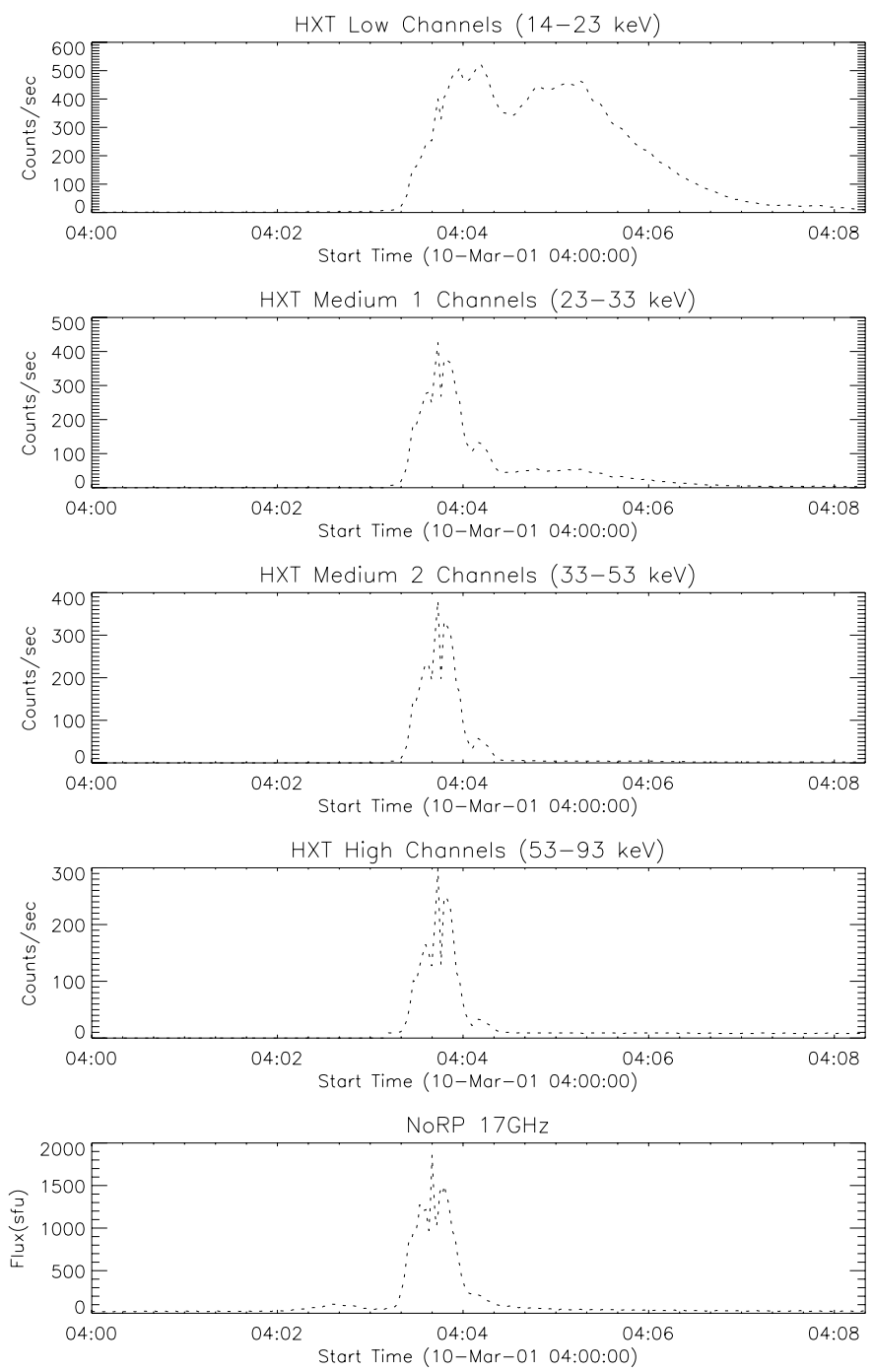

Figure 3. Yohkoh HXR lightcurves from the flare M6.7 of 1 March 2001 at L(14-23 keV), $\mathrm{M} 1(23-33 \mathrm{keV}), \mathrm{M} 2(33-53 \mathrm{keV})$ and $\mathrm{H}(53-93 \mathrm{keV})$. Last graph shows the $17 \mathrm{GHz} \mathrm{NoRP}$ temporal profile of the flare.

Uddin et al. (2004); Li, Ding \& Liu (2005) performed a very extensive study of the magnetic field of the active region 9368 and also studied the correlation of the HXR and MW emissions with the flaring region. They found that the shearing of the magnetic field was near to 80 degrees, which implies that a great amount of energy were stored in 
the magnetic field prior to the flare eruption. Non-linear force free field extrapolations of the magnetic fields revealed that the flaring region was dominated by low-lying magnetic field lines. Comparing this results with images taken by the SOHO-EIT instrument, we could see that the extrapolations were similar to the real magnetic field configuration, although the region was located near the limb.

The observed MW and HXR profiles may indicate some sort of seismicity induced by the flare, we decided to generate the time-distance plots for the flare and use the seismic holography to map the seismic region. We hve also used the boosting technique developed by Rajaguru et al. (2006) in order to emphasize the presence of the weak $6 \mathrm{mHz}$ seismic source that we detected. In the time-distance plot we have found a ridge over the azimuths $+135^{\circ}$ and $+225^{\circ}$ (Martínez-Oliveros et al. 2007b).

\section{Conclusions}

We have developed a multi-wavelength comparison technique for flares that make possible to assess the seismicity of a flare. This technique was successfully applied to two M-class solar flares showing the importance of the relation between trapped and precipitating electrons for the generation of seismic waves. The dynamical trapping model (Aschawanden 2004) gives a plausible explanation to emissions observed and the suddenness observed in these flares. The altitude of the flaring loops along with the dynamical trapping model seems to be another key ingredient for the generation of sun quakes, suggesting a possible more effective precipitation of electrons.

\section{Acknowledgements}

Juan Carlos Martínez-Oliveros thanks the Astronomical Society of Australia (ASA) and International Astronomical Union Symposium 247 (IAUS247) for travel support.

\section{References}

Aschwanden M.: 2004, Astrophys. J. 608, 554

Bastian, T.S., Benz, A.O, Gary, D.E: 1998, Annu. Rev. Astron. Astrophys., 36, 131

Besliu-Ionescu, D., Donea, A.-C., Cally, P. S. and Lindsey, C.: 2006, "Seismic Radiation from M-class Solar Flares," Beyond the Spherical Sun: a new era in helio-and asteroseismology, Proc. of SOHO-18/GONG-2006/HELAS I ed. B.Fleck (ESA Public.), CDROM, pp.67.1.

Ding, M. D.,Liu, Y., Yeh, C.-T., Li, J. P.: 2003, Astron. Astrophys. 403, 1151

Donea, A.-C., Beşliu-Ionescu, D., Lindsey, C. \& Zharkova, V. V. 2006, Solar Phys. 239, 113

Donea, A.-C., Braun, D. C \& Lindsey, C., 1999, Astrophys. J. 513, L143

Donea, A.-C., \& Lindsey, C., 2005 Astrophys. J.630, 1168.

Grechnev V.: 2003, Solar Phys. 213, 103

Kosovichev, A.G., Zharkova, V.V.,1998, Nature 393, 317

Kosovichev, A.G., 2006, Solar Phys. 238, 1

Li, J.P., Ding D.\& Liu, Y.: 2005, Solar Phys. 229, 115

Lindsey, C., \& Braun, D. C., 2000, Solar Phys.192, 261.

Liu, Y., Ding, M. D., and Fang, C: 2001, Astronom. J. 563, L169

Martinez-Oliveros, J.C., Moradi, H., Besliu-Ionescu, D.,Donea A.-C, Cally, P., Lindsey, C., 2007a, Solar Phys. 245, 121

Martinez-Oliveros, J.C., Moradi, H., D.,Donea A.-C 2007b, Solar Phys. submitted.

Moradi, H.; Donea, A.-C.; Lindsey, C.; Besliu-Ionescu, D.; Cally, P. S.,2007, Mon. Not. Roy. Astron. Soc. 374, 3, 1155

Rajaguru, S. P., Birch, A. C., Duvall, T. L., Jr., Thompson, M. J., Zhao, J.: 2006, Astrophys. J. 646,543

Uddin, W., Jain, R., Yoshimura, K., Chandra, R., Sakao, T., Kosugi, T., Joshi, A., Despande, M. R.: 2004, Solar Phys. 225, 325

Zharkova, V.V., \& Kobylinskii, V.A., 1993, Solar Phys. 143, 249.

Zharkova, V.V., \& Zharkov, S.I., 1993, Astrophys. J. 664, 573 